\title{
A largo plazo no existen diferencias en los resultados funcionales luego del tratamiento de cáncer de próstata localizado
}

In the long-term there are no differences in functional outcomes after treatment of localized prostate cancer

Matthew J. y cols., N Engl J Med 2013;368:436-45.

\section{Objetivos}

Comparar la funcionalidad intestinal, urinaria y sexual a largo plazo en pacientes con cáncer de próstata localizado sometidos a prostatectomía radical o radioterapia externa.

\section{Diseño, lugar y población}

Cohorte retrospectiva, utilizando la base de datos del Estudio de Resultados en Cáncer de Próstata (en inglés Prostate Cancer Outcomes Study, PCOS). Se incluyeron pacientes de seis ciudades de Estados Unidos, de entre 54 y 75 años con cáncer de próstata localizado, diagnosticados entre octubre 1994 y octubre 1995, que habían completado el seguimiento a los dos o cinco años y que habían recibido como tratamiento principal prostatectomía radical o radioterapia (con o sin deprivación androgénica). Los datos fueron recabados a través de cuestionarios que incluían aspectos clínicos, socio-demográficos y de calidad de vida en relación a la enfermedad al momento del diagnóstico y que luego fueron re-encuestados al año y a los 2, 5 y 15 años después del diagnóstico. Se utilizó el puntaje de propensión para controlar por la falta de asignación al azar del tratamiento.

\section{Resultados}

De los 3.533 pacientes de la cohorte PCOS se obtuvo una muestra final de 1.655 pacientes que incluía a la población de interés, 1.164 $(70,3 \%)$ con prostatectomía radical y $491(29,7 \%)$ con radioterapia externa. La tasa de respuesta en las encuestas a los 2, 5 y 15 años fue de $87,5,83,3$ y $60,3 \%$ respectivamente. A los 15 años de seguimiento, $322(27,7 \%)$ de los 1.164 pacientes con prostatectomía radical y 247 (50,3\%) de los 491 con radioterapia habían fallecido. Ver los resultados principales en la tabla 1.
Tabla 1. Resultados principales en ambos grupos a los 2, 5 y 15 años.

\begin{tabular}{l|c|c|c}
\multicolumn{1}{c|}{ Resultado } & $\begin{array}{c}\text { Prostatectomía } \\
\text { radical }\end{array}$ & Radioterapia & $\begin{array}{c}\text { OR ajustado } \\
\text { (IC 95\%) }\end{array}$ \\
\hline Incontinencia urinaria (\%) & & & \\
$\mathbf{2}$ años & 9,6 & 3,2 & $6,22(1,92 \mathrm{a} 20,29)$ \\
$\mathbf{5}$ años & 13,4 & 4,4 & $5,10(2,29 \mathrm{a} 11,36)$ \\
$\mathbf{1 5}$ años & 18,3 & 9,4 & $2,34(0,88 \mathrm{a} 6,23)$ \\
\hline Disfunción eréctil (\%) & & & \\
$\mathbf{2}$ años & 78,8 & 60,8 & $3,46(1,93 \mathrm{a} 6,17)$ \\
$\mathbf{5}$ años & 75,7 & 71,9 & $1,96(1,05$ a 3,63$)$ \\
$\mathbf{1 5}$ años & 87 & 93,9 & $0,38(0,12 \mathrm{a} 1,22)$ \\
\hline Urgencia intestinal (\%) & & & \\
$\mathbf{2}$ años & 13,6 & 34 & $0,39(0,22 \mathrm{a} 0,68)$ \\
$\mathbf{5}$ años & 16,3 & 31,3 & $0,47(0,26 \mathrm{a} 0,84)$ \\
$\mathbf{1 5}$ años & 21,9 & 35,8 & $0,98(0,45 \mathrm{a} 2,14)$ \\
\hline
\end{tabular}

$¥$ Odds ratio comparando prostatectomía radical con radioterapia, ajustado por registro, edad, función basal, raza o grupo étnico, grado del tumor, número de comorbilidades, nivel educativo y puntaje de propensión.

\section{Conclusiones}

A los 15 años de seguimiento no se observaron diferencias significativas en cuanto a resultados funcionales luego de la prostatectomía o radioterapia. No obstante, los pacientes tratados por cáncer de próstata localizado tuvieron un deterioro progresivo en todas las funciones estudiadas durante los 15 años de seguimiento.

Fuente de Financiamiento: Instituto Nacional del Cáncer. Los autores no refieren conflictos de interés.

\section{Comentario}

Los resultados funcionales luego de la prostatectomía radical están íntimamente relacionados con la consenvación de las bandeletas neurovasculares durante el procedimiento quirúrgico ${ }^{12}$. El presente artículo no informa acerca de la conservación o no de dichas estructuras, ni de la técnica quirúrgica utilizada. Varios estudios demuestran que la cirugía robótica tiene mejores resultados en cuanto a la continencia y potencia sexual (debido a la mejor conservación de las estructuras neurovasculares) que la cirugía convencional o laparoscópica².

Los autores revelan una pérdida de seguimiento considerable e informan una mortalidad del $27,7 \%$ para pacientes prostatectomizados y un $50,3 \%$ para irradiados. Sin embargo, no explican la causa de muerte ni el motivo de la pérdida de seguimiento, lo cual significa una fuente importante de sesgo a la hora de interpretar los resultados.

Más allá de las comparaciones funcionales, consideramos que el tratamiento a elegir implica la curación de una patología oncológica y, en este sentido, existen estudios observacionales que demuestran beneficios en favor de la prostatectomía radical ${ }^{3}$.

\section{Conclusiones de los comentadores}

Con respecto a los resultados funcionales no existen diferencias significativas entre la prostatectomia radical y la radioterapia a largo plazo, sin embargo, en el corto plazo sí existen y no es un dato menor ya que la calidad de vida del paciente dependerá de ello. Consideramos conveniente hacer hincapié en este aspecto a la hora de decidir un tratamiento, sin descuidar los resultados oncológicos. En la actualidad la incorporación de la tecnología robótica ha permitido mejorar los resultados funcionales de la prostatectomia radical manteniendo las altas tasas de curación.

Federico I. Tirapegui y Pablo Martinez. [ Servicio de Urología del Hospital Italiano de Buenos Aires

federico.tirapegui@ hospitalitaliano.org.ar, pablo.martinez@hospitalitaliano.org.ar ]

Tirapegui F; Martínez P. A largo plazo no existen diferencias en los resultados funcionales luego del tratamiento de cáncer de próstata localizado. Evid Act Pract Ambul. $2016 ; 19(2): 47$. Comentado de: Resnick, M, y col. Long-Term Functional Outcomes after Treatment for Localized Prostate Cancer. NEJM. 2013;368:436-445. PMID: 23363497.

\section{Referencias:}

1. Reeves F. y col. Preservation of the neurovascular bundles is associated with improved time to continence after radical prostatectomy but not long-term continence rates: results of a systematic review and meta-analysis. Eur Urol. 2015 Oct;68(4):692-704. doi: 10.1016/.eururo.2014.10.020. Epub 2014 Oct 29.

2. Ficarra V. y col. Systematic Review and Meta-analysis of Studies Reporting Potency Rates After Robot-assisted Radical Prostatectomy. Eur Urol. 2012; 62(3): 418-30.

3. Prasanna S y col. Comparative effectiveness of radical prostatectomy and radiotherapy in prostate cancer: observational study of mortality outcomes. BMJ 2014;348:g1502. 